\title{
Superfluid-quasicrystal in a Bose-Einstein condensate
}

\author{
Junpeng Hou, Haiping Hu, Kuei Sun, and Chuanwei Zhang* \\ Department of Physics, The University of Texas at Dallas, Richardson, Texas 75080-3021, USA
}

\begin{abstract}
Quasicrystal is a class of ordered structures defying conventional classification of solid crystals and may carry classically forbidden (e.g., 5-fold) rotational symmetries. In view of long-sought supersolids, a natural question is whether a superfluid can spontaneously form quasicrystalline order that is not possessed by the underlying Hamiltonian, forming "superfluid-quasicrystals". Here we show that a superfluid-quasicrystal stripe state with the minimal 5-fold rotational symmetry can be realized as the ground state of a Bose-Einstein condensate within a practical experimental scheme. There exists a rich phase diagram consisting of various superfluid-quasicrystal, supersolid, and plane-wave phases. Our scheme can be generalized for generating other higher-order (e.g., 7fold) quasicrystal states, and provides a platform for investigating such new exotic quantum matter.
\end{abstract}

Introduction. Quasicrystals exhibit exotic spatial patterns that are neither periodic as solid crystals (i.e., lack of translational symmetry) nor totally disordered (i.e., possession of long-range order) [1]. The Bragg diffraction peaks of quasicrystals possess rotational symmetries such as $5,7,8,9,10$-fold that are forbidden in classical crystalline orders $[1,2]$. Since its first report in Al-Mn and Al-Mn-Si alloys in 1984 [3], quasicrystal order has been studied and discovered in many different materials [4-10].

Supersolid, another exotic phase of matter, combines solid crystalline structure with superfluidity, where two continuous symmetries, namely, translational and $U(1)$ gauge, are spontaneously broken [11]. Supersolids were first predicted for helium almost 50 years ago [12, 13], and have recently been observed in cold atom experiments $[14,15]$, where a stripe phase with supersolid properties was generated and observed in a Bose-Einstein condensate (BEC) [14]. These great advances in the study of supersolids raise a natural question: is it possible to create a novel quantum matter where both superfluidity and quasicrystal orders coexist?

In this Letter, we address this important question by proposing a scheme to generate a stable quasicrystal ground state in a BEC. The experimental setup contains a 3D BEC confined in a 1D optical superlattice with quintuple wells (defines 5 pseudospin states), where neighboring wells are coupled by Raman assisted tunneling to generate an effective spin-orbit coupling (SOC) $[14,16]$ in the perpendicular plane. The scheme utilizes natural contact interaction and can realize quasicrystals with the minimum 5-fold rotational symmetry. In this new quantum state, the $U(1)$ gauge symmetry is spontaneously broken just as that in supersolid stripe phases $[14,16]$. However, the discrete translational symmetry, which is preserved in supersolids and leads to periodic density modulations in stripe phases $[14,16]$, has also been broken, leaving only specified rotational symmetry. A quasicrystal order with such rotational symmetry but no periodic spatial density modulation is spontaneously formed although the underlying Hamiltonian does not possess such order. Therefore we denote this quantum matter as 'superfluid-

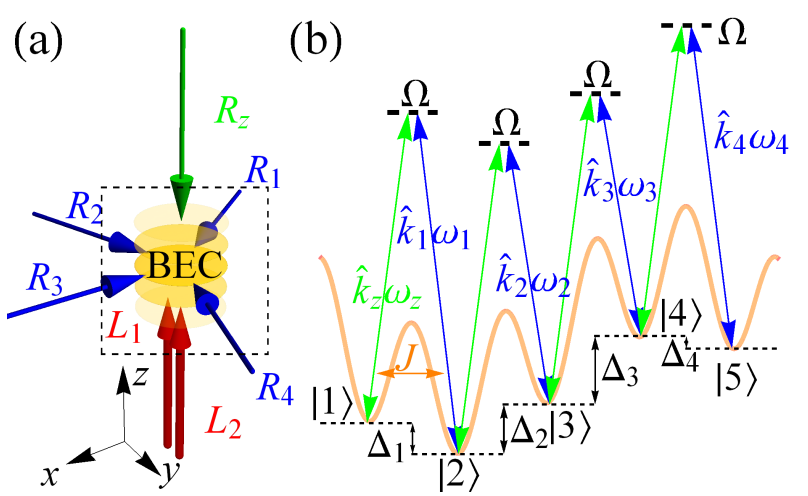

FIG. 1: (Color online) (a) Proposed experimental scheme for generating superfluid-quasicrystals with 5-fold rotational symmetry. The superlattice is generated by two optical lattices with different periods. A potential gradient can be generated using a magnetic field gradient. One Raman beam $R_{z}$ in the $z$-direction and four others $\left(R_{j}, j=1,2,3,4\right)$ in the $x-y$ plane generate the Raman coupling between neighboring wells. (b) SOC in one unit cell. Each Raman process only couples well $j$ to its adjacent neighbor $j+1$. The hopping between neighboring unit cells is irrelevant because of the large bias between $|1\rangle$ and $|5\rangle$. Only Raman-assisted inter-well tunneling in a unit cell is considered.

quasicrystal'. By tuning system parameters (e.g., Raman coupling strength, detuning, interaction, etc.), we show, through both variational ansatz analysis and direct simulation of mean field Gross-Pitaevskii equation (GPE), that there exists a rich phase diagram containing various superfluid-quasicrystals, supersolids, and plane-wave phases. Our scheme can be further extended to generate any $n$-order superfluid-quasicrystal phases. Our results may advance our understanding of both quasicrystals and superfluids and should provide an excellent platform for exploring many interesting properties of superfluidquasicrystals, a novel format of quantum matter.

Experimental scheme and Hamiltonian. We consider a 3D BEC confined in a tilted superlattice potential

$$
\begin{gathered}
V_{S L}(z)=V_{1} \sin ^{2}\left(k_{L 1} z\right)+V_{2} \sin ^{2}\left(k_{L 2} z+\phi_{12}\right)+\alpha_{z} z \text { (1) } \\
\text { along the } z \text {-direction [Fig. 1(a)] with } k_{L 2}=k_{L 1} / 5 \text {. Here }
\end{gathered}
$$


two lattices can come from the same laser source with the second lattice potential formed by two beams intersecting with an angle $\theta=2 \arcsin (1 / 5) \approx 23^{\circ}$. The linear potential $\alpha_{z} z$ can be realized with a magnetic field gradient. Note that this superlattice does not defy the definition for superfluid-quasicrystal because it only breaks the translational symmetry in the $z$-direction, while the spontaneous formation of (quasi)crystal order is on the $x-y$ plane. We denote five wells in each unit cell as five pseudospins and the effective couplings $\Omega$ between neighboring spins are induced by 5 Raman beams, one in the $z$ direction and four in the $x-y$ plane with designated wavevectors $\hat{k}_{j}$ [Fig. 1(b)]. We choose suitable parameters $\phi_{12}, \alpha_{z}$ such that the energy bias between neighboring wells $\left|\Delta_{j}\right| \gg J$ to avoid direct hopping ( $J$ is the bare tunneling rate without Raman coupling) and ||$\Delta_{j}|-| \Delta_{i}|| \gg \Omega$ for $i \neq j$ so that two neighboring wells are coupled only by one specific Raman pair.

The effective single particle Hamiltonian $H_{0}$ in the $x-y$ plane can be written as

$H_{0}=\sum_{j=1}^{5}\left[\frac{\left(\hat{p}-\hat{p}_{j}\right)^{2}}{2}+\delta_{j}\right]|j\rangle\langle j|+\sum_{j=1}^{4}\left(\frac{\Omega}{2}|j\rangle\langle j+1|+\right.$ h.c. $)$,

after a standard unitary transformation of the pseudospin phases to remove the spatial dependence of the Raman coupling [17]. Here we choose the units as $\hbar=k_{R}=m=$ 1 , where $k_{R}$ is recoil wavevector and $m$ is atomic mass. The energy unit $E_{R}=\hbar^{2} k_{R}^{2} / m=1 . \quad \delta_{j}$ is the detuning determined by the Raman transition. $\hat{p}_{j}$ satisfying $\hat{p}_{j}=\hat{p}_{j-1}+2 \hat{k}_{j-1}$ and $\hat{p}_{1}=-\frac{2}{5} \sum_{j=1}^{5}(5-j) \hat{k}_{j}[17]$. In order to generate good superfluid-quasicrystals, we consider a regular pentagon (all minima form an equilateral polygon) with $\hat{p}_{1}=(0,1), \hat{p}_{2}=(-\sqrt{5 / 8+\sqrt{5} / 8},(-1+$ $\sqrt{5}) / 4), \quad \hat{p}_{3}=(-\sqrt{5 / 8-\sqrt{5} / 8}, \quad(-1-\sqrt{5}) / 4)$, $\hat{p}_{4}=(\sqrt{5 / 8-\sqrt{5} / 8}, \quad(-1-\sqrt{5}) / 4)$, and $\hat{p}_{5}=$ $(\sqrt{5 / 8+\sqrt{5} / 8},(-1+\sqrt{5}) / 4)$ [see Fig.2(a)], which can be realized using $\hat{k}_{j}=\left(\hat{p}_{j+1}-\hat{p}_{j}\right) / 2(j=1,2,3,4)$ for four Raman lasers in the $x y$ plane.

For $\Omega=0$ and $\delta_{j}=0$ [Fig. 2(a)], five minima distribute over the vertexes of a regular pentagon. All spin components are uncoupled and only occupy one minimum. As a strong Raman coupling $\Omega=0.4$ is ramped on [Fig. 2(b)], the minima are coupled as an open boundary chain without coupling between head and tail. The spin components are mixed at each minimum and each well starts to merge with its adjacent neighbor. Because the locations of all minima [labelled as in Fig. 2(a)] are axisymmetric to the vector $\hat{p}_{3}$, the two uncoupled minima (the head and the tail) disappear first at certain critical value of $\Omega$ (we label the remaining minima as 2 to 4). Finally, the remaining three minima merge into one (minimum 3 ) approximately located at $(-0.207,-0.286)$

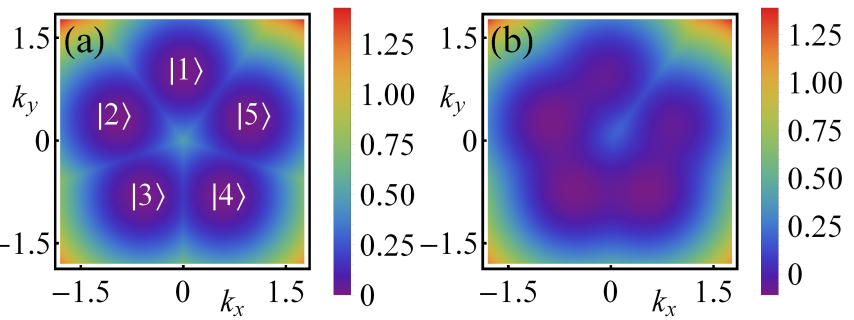

FIG. 2: (Color online) Single particle lowest band dispersion. (a) $\Omega=0$. The minima form a regular pentagon structure in the momentum space. (b) $\Omega=0.4$. The minima are strongly coupled and all spin components are mixed. Wells 1 and 5 are uncoupled and a barrier between them can be clearly observed. The five minima are axisymmetric to $\hat{p}_{3}$ for any $\Omega$.

[17] when $\Omega$ is extremely strong and its location is still along the same line as $\hat{p}_{3}$.

Phase diagram. We now study new quantum phases emerging from interactions between atoms, which can be described by the GPE under the mean-field approximation with energy density

$$
\epsilon=\int \frac{d \hat{r}}{V}\left[\psi^{\dagger} H_{0} \psi+\frac{c_{0}}{2} n_{i}^{2}+\frac{c_{2}}{2} \sum_{i=1}^{4} n_{i} n_{i+1}\right],
$$

where $\psi$ is the 5-component spinor wavefunction, $n_{i}=$ $\psi_{i}^{\dagger} \psi_{i}$ is the density for the spin component $i, c_{0}$ and $c_{2} \approx \frac{J}{\Delta} c_{0}$ are density interaction for the same and neighboring spins, respectively. For realistic parameters, $J / \Delta \sim 1 / 20$, the neighboring spin interaction can be ignored [14] and this is a crucial condition for realizing superfluid-quasicrystals or supersolids in experiments. The wavefunction is normalized by the average atomic density as $V^{-1} \int d \hat{r} \psi^{\dagger} \psi=\bar{n}$ with $V$ being the system volume. We obtain the ground state using both variational ansatz analysis and direct numerical simulation of the GPE, and they agree well.

The general form of the variational ansatz is

$$
\psi=\sqrt{\bar{n}} \sum_{j=1}^{5} C_{j} e^{i \hat{k}_{m, j} \cdot \hat{r}} \xi_{j},
$$

where $C_{j}$ are complex numbers satisfying normalization relation $\sum_{j}\left|C_{j}\right|^{2}=1, \hat{k}_{m, j}$ denotes each minimum in momentum space and $\xi_{j}$ are the spinor part of wavefunction $\xi_{j}=\left(\cos \alpha_{j} \cos \beta_{j} \cos \gamma_{j}, \cos \alpha_{j} \cos \beta_{j} \sin \gamma_{j}, \sin \alpha_{j}\right.$, $\left.\cos \alpha_{j} \sin \beta_{j} \sin \eta_{j}, \quad \cos \alpha_{j} \sin \beta_{j} \cos \eta_{j}\right)^{T}$. We assume $\xi_{1, j}=\xi_{5,6-j}, \xi_{2, j}=\xi_{4,6-j}\left(\xi_{i, j}\right.$ stands for the $j$ th component of spinor $\left.\xi_{i}\right)$, and $\hat{k}_{m, 1}\left(\hat{k}_{m, 2}\right)$ and $\hat{k}_{m, 5}\left(\hat{k}_{m, 4}\right)$ are axisymmetric to vector $\hat{k}_{m, 3}$ based on the symmetry of the Hamiltonian. Generally it is challenging to optimize the energy density functional with so many variables. However, in the weak interaction region $\bar{n} c_{0} \ll 1$, the BEC wavefunction at each band minimum is quite close to the single particle spinor wavefunction, which can thus be used to fix $\xi_{j}$ for the variational calculation. Simi- 

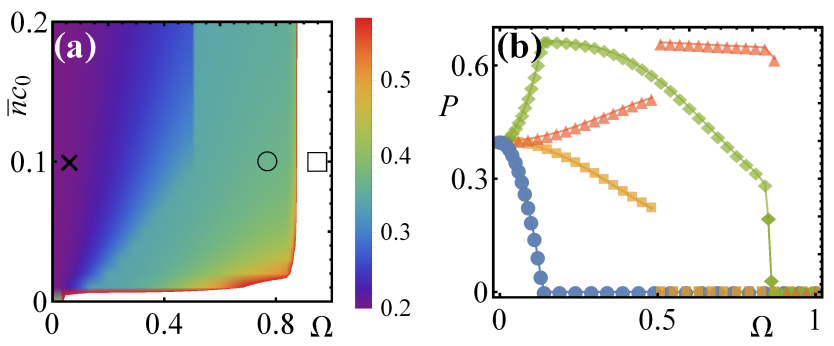

FIG. 3: (Color online) (a) Phase diagram from the variational ansatz analysis when all the detunings $\delta_{j}$ are set to be zero. The color represents the value of $\left|C_{3}\right|^{2}$. The white region is the plane-wave phase. The three symbols (cross, circle, and square stand for $\Omega=0.06,0.77$ and 0.95 ) along $\bar{n} c_{0}=0.1$ are examples for superfluid-quasicrystal, supersolid, and planewave phases. (b) Phase transitions between different phases. The blue circles (green rhombus) and orange squares (red triangles) show how $\left|C_{1}\right|^{2}+\left|C_{5}\right|^{2}\left(\left|C_{2}\right|^{2}+\left|C_{4}\right|^{2}\right)$ varies with Raman coupling for $\bar{n} c_{0}=0.01$ and 0.2 , respectively.

lar method was used previously for studying spin-1 spinorbit coupled BEC, which gives all phases as those in full variational calculation, although the phase boundary may be slightly different for stronger interaction [18]. We also find that $\left|C_{1}\right|=\left|C_{5}\right|$ and $\left|C_{2}\right|=\left|C_{4}\right|$ hold in weak interaction cases. The ground state energy is degenerate with respect to relative phases between $C_{j}$ and the system spontaneously chooses one set of relative phases for the superfluid-quasicrystal and supersolid stripe phases.

In Fig. 3(a), we plot the phase diagram with respect to the interaction strength $\bar{n} c_{0}$ and Raman coupling strength $\Omega$ obtained from the variational ansatz calculation, where the color shows the occupied probability $\left|C_{3}\right|^{2}$ at the momentum minimum 3. At a finite $\Omega, 3$ has the lowest energy, therefore atoms only occupy 3 without interaction, leading to a plane-wave phase. On the other hand, a strong density-density interaction prefers the equal occupation of all minima. Therefore the competition between Raman coupling and interaction may render different phases, as shown in Fig. 3(a).

In the small $\Omega$ region, all five minima are equally populated with the same probability $1 / 5$ due to interaction, forming a superfluid-quasicrystal [cross in 3(a)]. This new quantum matter is confirmed by the real and momentum space density distributions [Fig. 4(a,b)] obtained from the GPE simulation in a harmonic trap. We see the distribution in the real space is indeed in lack of translational symmetry, while in momentum space five equally populated peaks form a regular pentagon with each vertex designated as $\hat{p}_{j}$, showing the 5 -fold rotational symmetry of the superfluid-quasicrystal phase. Note that the harmonic trap breaks the degeneracy of the ground state and fixes the relative phases between $C_{j}$.

With the increase in $\Omega$, the occupation of five minima changes to three, leading to a supersolid stripe phase [circle in Fig. 3(a)], where $\left|C_{3}\right|^{2}$ increases to $\sim 0.35$. The
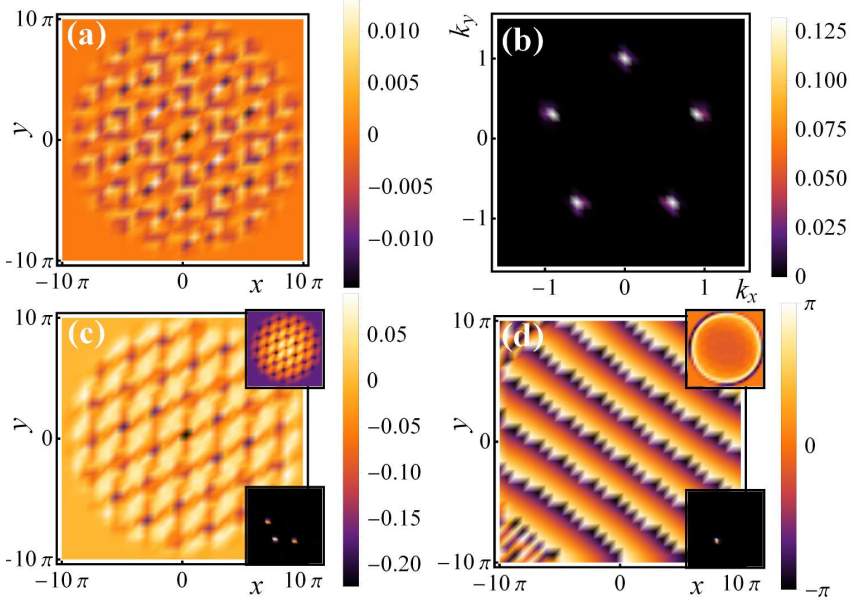

FIG. 4: (Color online) (a)[(b)] Real-space (momentum-space) distribution for the superfluid-quasicrystal phase from GPE simulation. The parameters are the same as the black cross marked in Fig. 3(a). The five minima in momentum space are evenly populated. (c) Spatial distribution for a supersolid phase [parameters chosen as black circle in Fig. 3(a)] with three minima populated. The top and bottom insets show the real-space density of state $|3\rangle$ and the momentum-space distribution respectively. (d) Phase distribution for state $|3\rangle$ at a nonzero momentum plane-wave phase, and the insets give real (top) and momentum (bottom) space distributions. The parameters correspond to the black square in Fig. 3(a). All real-space density distributions (including following panels) are obtained through subtracting the real-space density at zero Raman coupling from those of finite $\Omega$ to rule out the large density variation across the harmonic trap. The plots are rescaled over the average density in the trap. The total momentum-space distribution is the direct summation of that for each pseudospin component.

resulting real and momentum space density distributions from GPE are shown in Fig. 4(c). Here a clear translational symmetry in the real space is observed. In the momentum space distribution (bottom inset), three minima are occupied unevenly and minimum 3 has a larger weight. Here $\Omega$ is quite large and the spin components in each minimum are mixed. Consequently, the spatial distribution of state $|3\rangle$ exhibits clear density modulation (top inset). For a very large $\Omega$, all minima merge to one and the system enters a plane-wave phase [square in 3(a)]. In Fig. 4(d), we plot its phase distribution obtained from GPE, which shows a stripe pattern as expected. The overall real space density distribution (top inset) is a Gaussian-type wavepacket and the BEC occupies one point in the momentum space (bottom inset).

We characterize the transition between these phases in Fig. 3(b), where we plot the populations $P_{15}=\left|C_{1}\right|^{2}+$ $\left|C_{5}\right|^{2}$ and $P_{24}=\left|C_{2}\right|^{2}+\left|C_{4}\right|^{2}$ with respect to $\Omega$ for two different interaction strengths $\bar{n} c_{0}=0.01$ and 0.2 . In the weak interaction case $\bar{n} c_{0}=0.01, P_{15}$ smoothly decreases to 0 and $P_{24}$ has a sharp turn at certain $\Omega$, showing a second-order phase transition from superfluid- 

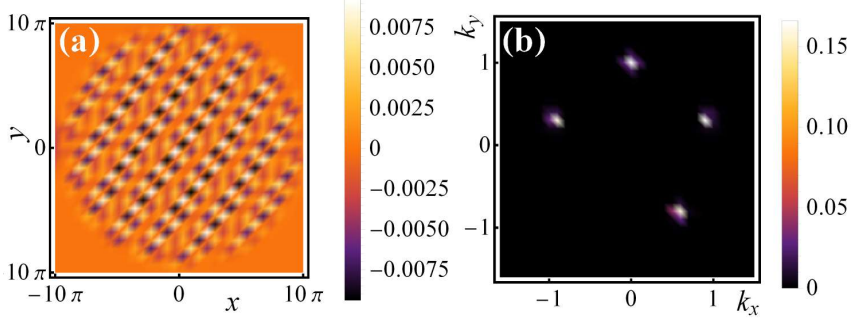

FIG. 5: (Color online) (a)[(b)] Real-space (momentum-space) distribution for a supersolid phase when one minimum is knocked off with a small detuning $\delta_{3} \sim 0.01 E_{R}$ in the superfluid-quasicrystal phase in Fig. 4(a,b).

quasicrystal to supersolids. This occurs when the energy bias between minima 1 and 2 with increasing $\Omega$ is larger than the interaction energy cost. For the strong interaction $\bar{n} c_{0}=0.2, P_{15}\left(P_{24}\right)$ shows a sudden drop at $\Omega \sim 0.5$, showing a first-order phase transition at the point where single particle five minima merge into three. Before the transition, the interaction energy cost is so strong that $P_{15}$ is always nonzero. Around $\Omega \sim 0.8$, the three minima merge into one and $P_{24}$ also suddenly drops to zero, showing a first order phase transition to the plane wave phase.

In addition to varying $\Omega$, we may also adjust detuning $\delta_{j}$ to change the relative population of the minima. In Fig. 5(a,b), we plot the real and momentumspace distribution from GPE by adding a small detuning $\delta_{3} \sim 0.01 E_{R}$ in spin state $|3\rangle$ for the superfluidquasicrystal state in Fig 4(a). We see that the minimum 3 is now knocked out and the translational symmetry is restored. The BEC becomes a supersolid with exotic real-space distribution because it is still populated on four of five vertexes of a regular pentagon. The interplay among Raman coupling, detuning, and interaction leads to a rich phase diagram and hence enables the designing and engineering of new superfluid-quasicrystal and supersolid phases.

Experimental realization and detection. The experimental realization of our scheme is in the same spirit as recent experimental reports on observing supersolid stripe phases $[14,16]$. Consider $N=10^{5}{ }^{23} \mathrm{Na}$ atoms confined in a super-lattice with five wells in one unit cell and the condensate is initially split into each well equally with an average density around $\bar{n}=0.5 \times 10^{14} \mathrm{~cm}^{-3}$. We choose $k_{R}=1064 \mathrm{~nm}$ and thus, $E_{R}=7672 \mathrm{~Hz}$, for Raman lasers. The Raman coupling strength $\Omega$ can be tuned as low as $300 \mathrm{~Hz}[14,16]$, that is, $\Omega \lesssim 0.08 E_{R}$, which well resides in the superfluid-quasicrystal region. In real experiments, $\left(c_{0}-c_{2}\right) / c_{0} \approx 1$, therefore the neighboring-spin interaction term can be neglected. The same spin density interaction strength can be evaluated with $c_{0}=4 \pi \hbar^{2} a_{s} / m$, where $a_{s}$ is the two-body scattering length. Taking $a_{s}=50 a_{0}$ ( $a_{0}$ is Bohr radius) [19, 20], we have $\bar{n} c_{0} \approx 0.1 E_{R}$, which is sufficiently strong for the observation of superfluid-quasicrystals, although a larger density $\bar{n}$ or scattering length $a_{s}$ (tuned by Feshbach resonance) yields a larger parameter region (Fig. 3) and is better for the observation of superfluid-quasicrystal phases.

In experiments, the superfluid-quasicrystal phases may be observed in the time-of-flight (TOF) image, where five equally populated peaks are formed in the momentum space at designated positions [Fig. 4(b)]. By measuring $P_{15}$ and $P_{24}$ in TOF, the quantum phase transition between different phases in Fig. 3(b) can be detected. Another way to observe the superfluid-quasicrystal phases is using Bragg scattering, similar to that for supersolids [14], where the Bragg diffraction patterns for superfluidquasicrystals should give peaks possessing 5 -fold rotational symmetry [2].

Discussion and Conclusion. Our proposed experimental setup can be straightforwardly generalized for realizing other superfluid-quasicrystal phases with higher order rotational symmetry, such as $n=7$, where 7 Raman lasers are needed with a similar experimental setup for 7 wells in a superlattice. Similar idea can also be applied to generate superfluid-quasicrystal phases in a spin-orbit coupled BEC with atomic hyperfine state pseudospins [21-33], where supersolid stripe phases have been proposed [34-44] for both $1 \mathrm{D}$ and 2D SOC, but have not been observed in experiments. Note that our proposed scheme for superfluid-quasicrystals requires five almost degenerate band minima for five different spins to generate a regular pentagon in the momentum space. In experiments, an effective 2D SOC (not exactly Rashba) has been experimentally realized recently [31-33] by coupling three spin states at three degenerate band minima, although the resulting band minimum path in the lowest band is not a flat ring as expected from a Rashba SOC. In our scheme, no flat Rashba ring is needed and 5 Raman lasers with suitable wavevectors and polarizations are chosen such that the effective band minima are formed at $\hat{p}_{j}$ for a regular pentagon. The crucial difficulty comes from the interaction that is almost isotropic between any spin states. This difficulty may be resolved using ${ }^{133} \mathrm{Cs}$ atoms [45], where the interaction may be tuned by Feshbach resonance to favor the equal occupation of five minima in the momentum space, instead of the plane-wave at one minimum.

In summary, we have proposed a scheme for realizing superfluid-quasicrystal stripe phases using a BEC in a 1D quintuple-well optical superlattice with Ramanassisted tunneling. Through variational and GPE analysis, we show there is a rich phase diagram containing superfluid-quasicrystals, supersolids, plane-wave phases, and their phase transitions. Our proposed experimental setup should lay out a platform for future theoretical and experimental investigations of such exotic novel quantum matter. 
Acknowledgments: This work is supported by AFOSR (FA9550-16-1-0387), NSF (PHY-1505496), and ARO (W911NF-17-1-0128).

* Corresponding author.

Email: chuanwei.zhang@utdallas.edu

[1] J. Christian, Neutron and Synchrotron Radiation for Condensed Matter Studies, Springer Berlin Heidelberg, 1994.

[2] D. Levine and P. J. Steinhardt, Quasicrystals: A New Class of Ordered Structures, Phy. Rev. Lett. 53, 2477 (1984).

[3] D. Shechtman, I. Blech, D. Gratias, and J. W. Cahn, Metallic Phase with Long-Range Orientational Order and No Translational Symmetry, Phy. Rev. Lett. 53, 1951 (1984).

[4] E. Maciá, The role of aperiodic order in science and technology, Rep. Prog. Phys. 69, 397 (2006).

[5] B. Freedman, R. Lifshitz, J. W. Fleischer \& M. Segev, Phason dynamics in nonlinear photonic quasicrystals, Nat. Mater. 6, 776 (2007).

[6] K. Barkan, H. Diamant, and R. Lifshitz, Stability of quasicrystals composed of soft isotropic particles, Phy. Rev. B 83, 172201 (2011).

[7] N. A. Wasio, R. C. Quardokus, R. P. Forrest, C. S. Lent, S. A. Corcelli, J. A. Christie, K. W. Henderson \& S. Alex Kandel, Self-assembly of hydrogen-bonded two-dimensional quasicrystals, Nature (London) 507, 86 (2014).

[8] K. Nagao, T. Inuzuka, K. Nishimoto, and K. Edagawa, Experimental Observation of Quasicrystal Growth, Phy. Rev. Lett. 115, 075501 (2015).

[9] J. I. Urgel, D. Écija, G. Lyu, R. Zhang, C.-A. Palma, W. Auwärter, N. Lin \& J. V. Barth, Quasicrystallinity expressed in two-dimensional coordination networks, Nat. Chem. 8, 657 (2016).

[10] L. Bindi, P. J. Steinhardt, N. Yao, P. J. Lu, Natural Quasicrystals, Science 324, 1306 (2009).

[11] M. Boninsegni and N. V. Prokofv, Supersolids: What and where are they? Rev. Mod. Phys. 84, 759 (2012).

[12] D.J Thouless, The flow of a dense superfluid, Ann. Phys. 52, 403 (1971).

[13] A. F. Andreev and I. M. Lifshitz, Quantum Theory of Defects in Crystals, Sov. Phys. JETP 29, 1107 (1971).

[14] J.-R. Li, J. Lee, W. Huang, S. Burchesky, B. Shteynas, F. Ç. Top, A. O. Jamison, and W. Ketterle, A stripe phase with supersolid properties in spin-orbit-coupled BoseEinstein condensates, Nature (London) 543, 91 (2017).

[15] J. Léonard, A. Morales, P. Zupancic, T. Esslinger \& T. Donner, Supersolid formation in a quantum gas breaking a continuous translational symmetry, Nature (London) 543, 87 (2017).

[16] J. Li, W. Huang, B. Shteynas, S. Burchesky, F. Top, E. Su, J. Lee, A. O. Jamison, and W. Ketterle, SpinOrbit Coupling and Spin Textures in Optical Superlattices, Phy. Rev. Lett. 117, 185301 (2016).

[17] Supplementary materials, see supplementary materials for details.

[18] X.-W. Luo, K. Sun, C. Zhang, Spin-tensor-momentumcoupled Bose-Einstein condensates, Phys. Rev. Lett.
119, 193001 (2017).

[19] F. A. van Abeelen and B. J. Verhaar, Determination of collisional properties of cold $\mathrm{Na}$ atoms from analysis of bound-state photoassociation and Feshbach resonance field data, Phy. Rev. A 59, 578 (1999).

[20] S. Knoop, T. Schuster, R. Scelle, A. Trautmann, J. Appmeier, M. K. Oberthaler, E. Tiesinga, and E. Tiemann, Feshbach spectroscopy and analysis of the interaction potentials of ultracold sodium, Phy. Rev. A 83, 042704 (2011).

[21] Y.-J. Lin, K. Jiménez-García, and I. B. Spielman, Spinorbit-coupled Bose-Einstein condensates, Nature (London) 471, 83 (2011).

[22] J.-Y. Zhang, S.-C. Ji, Z. Chen, L. Zhang, Z.-D. Du, B. Yan, G.-S. Pan, B. Zhao, Y.-J. Deng, H. Zhai, S. Chen, and J.-W. Pan, Collective Dipole Oscillations of a Spin-Orbit Coupled Bose-Einstein Condensate, Phys. Rev. Lett. 109, 115301 (2012).

[23] C. Qu, C. Hamner, M. Gong, C. Zhang, and P. Engels, Observation of Zitterbewegung in a spin-orbit-coupled Bose-Einstein condensate, Phys. Rev. A 88, 021604(R) (2013).

[24] A. J. Olson, S.-J. Wang, R. J. Niffenegger, C.-H. Li, C. H. Greene, and Y. P. Chen, Tunable Landau-Zener transitions in a spin-orbit-coupled Bose-Einstein condensate, Phys. Rev. A 90, 013616 (2014).

[25] C. Hamner, C. Qu, Y. Zhang, J. Chang, M. Gong, C. Zhang, and P. Engels, Dicke-type phase transition in a spin-orbit-coupled Bose-Einstein condensate, Nat. Commun. 5, 4023 (2014).

[26] P. Wang, Z.-Q. Yu, Z. Fu, J. Miao, L. Huang, S. Chai, H. Zhai, and J. Zhang, Spin-Orbit Coupled Degenerate Fermi Gases, Phys. Rev. Lett. 109, 095301 (2012).

[27] L. W. Cheuk, A. T. Sommer, Z. Hadzibabic, T. Yefsah, W. S. Bakr, and M. W. Zwierlein, Spin-Injection Spectroscopy of a Spin-Orbit Coupled Fermi Gas, Phys. Rev. Lett. 109, 095302 (2012).

[28] R. A. Williams, M. C. Beeler, L. J. LeBlanc, K. JiménezGarcía, and I. B. Spielman, Raman-Induced Interactions in a Single-Component Fermi Gas Near an $s$-Wave Feshbach Resonance, Phys. Rev. Lett. 111, 095301 (2013).

[29] N. Q. Burdick, Y. Tang, and B. L. Lev, Long-Lived SpinOrbit-Coupled Degenerate Dipolar Fermi Gas, Phys. Rev. X 6, 031022 (2016).

[30] B. Song, C. He, S. Zhang, E. Hajiyev, W. Huang, X.-J. Liu, and G.-B. Jo, Spin-orbit-coupled two-electron Fermi gases of ytterbium atoms, Phys. Rev. A 94, 061604(R) (2016).

[31] L. Huang, Z. Meng, P. Wang, P. Peng, S.-L. Zhang, L. Chen, D. Li, Q. Zhou \& J. Zhang, Experimental realization of two-dimensional synthetic spin-orbit coupling in ultracold Fermi gases, Nat. Phys. 12, 540 (2016).

[32] Z. Meng, L. Huang, P. Peng, D. Li, L. Chen, Y. Xu, C. Zhang, P. Wang, and J. Zhang, Experimental Observation of a Topological Band Gap Opening in Ultracold Fermi Gases with Two-Dimensional Spin-Orbit Coupling, Phys. Rev. Lett. 117, 235304 (2016).

[33] Z. Wu, L. Zhang, W. Sun, X.-T. Xu, B.-Z. Wang, S.-C. Ji, Y. Deng, S. Chen, X.-J. Liu, J.-W. Pan, Realization of two-dimensional spin-orbit coupling for Bose-Einstein condensates, Science 354, 83 (2016).

[34] T. D. Stanescu, B. Anderson, and V. Galitski, Spinorbit coupled Bose-Einstein condensates, Phys. Rev. A 78, 023616 (2008). 
[35] C. Wu, I. Mondragon-Shem, and X.-F. Zhou, Unconventional Bose-Einstein Condensations from Spin-Orbit Coupling, Chin. Phys. Lett. 28, 097102 (2011).

[36] C. Wang, C. Gao, C.-M. Jian, and H. Zhai, Spin-orbit coupled spinor Bose-Einstein condensates, Phys. Rev. Lett. 105, 160403 (2010).

[37] T.-L. Ho and S. Zhang, Bose-Einstein condensates with spin-orbit interaction, Phys. Rev. Lett. 107, 150403 (2011).

[38] Y. Li, L. Pitaevskii, and S. Stringari, Quantum tricriticality and phase transitions in spin-orbit coupled BoseEinstein condensates, Phys. Rev. Lett. 108, 225301 (2012).

[39] Y. Zhang, L. Mao, and C. Zhang, Mean-field dynamics of spin-orbit coupled Bose-Einstein condensates, Phys. Rev. Lett. 108, 035302 (2012).

[40] H. Hu, B. Ramachandhran, H. Pu, and X.-J. Liu, Spinorbit coupled weakly interacting Bose-Einstein condensates in harmonic traps, Phys. Rev. Lett. 108, 010402 (2012).

[41] T. Ozawa and G. Baym, Stability of ultracold atomic bose condensates with rashba spin-orbit coupling against quantum and thermal fluctuations, Phys. Rev. Lett. 109, 025301 (2012).

[42] K. Sun, C. Qu, Y. Xu, Y. Zhang, and C. Zhang, Interacting spin-orbit-coupled spin-1 Bose-Einstein condensates, Phys. Rev. A 93, 023615 (2016).

[43] Z.-Q. Yu, Phase transitions and elementary excitations in spin-1 Bose gases with Raman-induced spin-orbit coupling, Phys. Rev. A 93, 033648 (2016).

[44] G. Martone, F. Pepe, P. Facchi, S. Pascazio, and S. Stringari, Tricriticalities and quantum phases in spinorbit-coupled spin-1 bose gases, Phys. Rev. Lett. 117, 125301 (2016).

[45] L. W. Clark, L.-C. Ha, C.-Y. Xu, and C. Chin, Quantum Dynamics with Spatiotemporal Control of Interactions in a Stable Bose-Einstein Condensate, Phys. Rev. Lett. 115, 155301 (2015). 


\section{Supplementary materials}

\section{Single particle Hamiltonian for spin-orbit-coupling}

We derive the effective single-particle Hamiltonian for our pseudospin system with $n=5$ and the method can be generalized to arbitrary $n$. Results for $n=2$ have been studied with great details in Ref. [16]. The single particle Hamiltonian consists of two parts: the superlattice potential $V_{S L}$ in the $z$-direction and the Raman coupling.

Consider a superlattice with a tilted potential along the $z$ direction

$$
H_{S L}=\frac{\hat{p}^{2}}{2}+\frac{p_{z}^{2}}{2}+V_{1} \sin ^{2}\left(k_{L 1} z\right)+V_{2} \sin ^{2}\left(k_{L 2} z+\phi_{12}\right)+\alpha_{z} z
$$

where $k_{L 1}=\pi / d, k_{L 2}=5 \pi / d$, and $d$ is the period for the long lattice. In the tight-binding limit,

$$
\begin{aligned}
H_{S L}= & \frac{\hat{p}^{2}}{2}+\left(\Delta_{1}+\Delta_{2}\right) \sum_{m}\left|\Psi_{1, m}\right\rangle\left\langle\Psi_{1, m}\left|+\Delta_{2} \sum_{m}\right| \Psi_{2, m}\right\rangle\left\langle\Psi_{2, m}\right| \\
& -\Delta_{3} \sum_{m}\left|\Psi_{4, m}\right\rangle\left\langle\Psi_{4, m}\left|-\left(\Delta_{4}+\Delta_{3}\right) \sum_{m}\right| \Psi_{5, m}\right\rangle\left\langle\Psi_{5, m}\right| \\
& +J \sum_{m} \sum_{j=1}^{4}\left(\left|\Psi_{j, m}\right\rangle\left\langle\Psi_{j+1, m}\right|+\text { h.c. }\right)+\sum_{m} \sum_{l=1}^{5} \sum_{j=1}^{5}\left(J_{l, j}\left|\Psi_{l, m}\right\rangle\left\langle\Psi_{j, m+1}\right|+\text { h.c. }\right),
\end{aligned}
$$

where $\left|\Psi_{j, m}\right\rangle$ is the onsite wavefunction of well $j$ in the $m$-th unit cell. Well 3 is set as the reference of zero-energy and $\Delta_{j}$ is the energy difference between wells $j$ and $j+1$. Hereafter we neglect the coupling $J_{l, j}$ between adjacent unit cells because there is no Raman assisted tunneling between wells in different unit cells. Since $J \ll \Delta_{j}$, the wavefunctions can be expanded to the first-order

$$
\begin{aligned}
& |1, m\rangle=\left|\Psi_{1, m}\right\rangle+\frac{J}{\Delta_{1}}\left|\Psi_{2, m}\right\rangle,|2, m\rangle=\left|\Psi_{2, m}\right\rangle-\frac{J}{\Delta_{1}}\left|\Psi_{1, m}\right\rangle+\frac{J}{\Delta_{2}}\left|\Psi_{3, m}\right\rangle \\
& |3, m\rangle=\left|\Psi_{3, m}\right\rangle-\frac{J}{\Delta_{2}}\left|\Psi_{2, m}\right\rangle+\frac{J}{\Delta_{3}}\left|\Psi_{4, m}\right\rangle,|4, m\rangle=\left|\Psi_{4, m}\right\rangle-\frac{J}{\Delta_{3}}\left|\Psi_{3, m}\right\rangle+\frac{J}{\Delta_{4}}\left|\Psi_{5, m}\right\rangle,|5, m\rangle=\left|\Psi_{5, m}\right\rangle-\frac{J}{\Delta_{4}}\left|\Psi_{4, m}\right\rangle .
\end{aligned}
$$

The four Raman couplings are

$$
V_{\text {Raman }, j}=\Omega_{j} \cos \left(k_{z} z+\hat{k}_{j} \cdot \hat{r}-\delta_{R, j} t\right),
$$

where $k_{z}=5 \pi /(2 d)$. Expanding $H_{S L}$ under above perturbed basis, we obtain

$$
\begin{aligned}
H_{S L}= & \frac{\hat{p}^{2}}{2}+\left(\Delta_{1}+\Delta_{2}\right) \sum_{m}(|1, m\rangle\langle 1, m|)+\Delta_{2} \sum_{m}|2, m\rangle\langle 2, m| \\
& -\left(\Delta_{4}+\Delta_{3}\right) \sum_{m}|5, m\rangle\left\langle 5, m\left|-\Delta_{3} \sum_{m}\right| 4, m\right\rangle\langle 4, m| \\
+ & \sum_{\hat{p}, \hat{p}^{\prime}}|\hat{p}\rangle\left(\sum_{n^{\prime}} \sum_{l=1}^{5} \sum_{j=1}^{5}|l, m\rangle\left\langle l, m\left|\left\langle\hat{p}\left|\Omega_{j} \cos \left(k_{z} z+\hat{k_{j}} \cdot \hat{r}-\delta_{j} t\right)\right| \hat{p}^{\prime}\right\rangle\right| j, m\right\rangle\langle j, m|\right)\left\langle\hat{p}^{\prime}\right|,
\end{aligned}
$$

where

$$
\begin{aligned}
\left\langle 1, m\left|\cos \left(k_{z}\left(z-z_{m}\right)\right)\right| 2, m\right\rangle & =-\frac{J}{\Delta_{1}},\left\langle 2, m\left|\cos \left(k_{z}\left(z-z_{m}\right)\right)\right| 3, m\right\rangle=-\frac{J}{\Delta_{2}}, \\
\left\langle 3, m\left|\cos \left(k_{z}\left(z-z_{m}\right)\right)\right| 4, m\right\rangle & =\frac{J}{\Delta_{3}},\left\langle 4, m\left|\cos \left(k_{z}\left(z-z_{m}\right)\right)\right| 5, m\right\rangle=\frac{J}{\Delta_{4}} \\
\left\langle 1, m\left|\sin \left(k_{z}\left(z-z_{m}\right)\right)\right| 2, m\right\rangle & =\frac{J}{\Delta_{1}},\left\langle 2, m\left|\sin \left(k_{z}\left(z-z_{m}\right)\right)\right| 3, m\right\rangle=-\frac{J}{\Delta_{2}} \\
\left\langle 3, m\left|\sin \left(k_{z}\left(z-z_{m}\right)\right)\right| 4, m\right\rangle & =-\frac{J}{\Delta_{3}},\left\langle 4, m\left|\sin \left(k_{z}\left(z-z_{m}\right)\right)\right| 5, m\right\rangle=\frac{J}{\Delta_{4}} \\
\left\langle j, m\left|\cos \left(k_{z}\left(z-z_{m}\right)\right)\right| j, m\right\rangle & =\sin \left(\frac{\pi}{2} j\right),\left\langle j, m\left|\sin \left(k_{z}\left(z-z_{m}\right)\right)\right| j, m\right\rangle=-\cos \left(\frac{\pi}{2} j\right),
\end{aligned}
$$


up to the first order, $z_{m}=m d$ is the position of the 1 -st well in the $m$-th unit cell. With these relations, the Raman potential can be reformulated as

$$
\begin{aligned}
& \sum_{l=1}^{5} \sum_{j=1}^{5}|l, m\rangle\left\langle l, m\left|\left\langle\hat{p}\left|\Omega \cos \left(k_{z} z+\hat{k_{j}} \cdot \hat{r}-\delta_{j} t\right)\right| \hat{p}^{\prime}\right\rangle\right| j, m\right\rangle\langle j, m| \\
= & \Omega_{j} \cos \phi_{m, j}(|1, m\rangle\langle 1, m|-| 3, m\rangle\langle 3, m|+| 5, m\rangle\langle 5, m|) \\
& +\Omega_{j} \cos \phi_{m, j}\left(-\frac{J}{\Delta_{1}}|1, m\rangle\left\langle 2, m\left|-\frac{J}{\Delta_{2}}\right| 2, m\right\rangle\left\langle 3, m\left|+\frac{J}{\Delta_{3}}\right| 3, m\right\rangle\left\langle 4, m\left|+\frac{J}{\Delta_{4}}\right| 4, m\right\rangle\langle 5, m|\right) \\
& +\Omega_{j} \sin \phi_{m, j}(|2, m\rangle\langle 2, m|-| 4, m\rangle\langle 4, m|) \\
& +\Omega_{j} \sin \phi_{m, j}\left(\frac{J}{\Delta_{1}}|1, m\rangle\left\langle 2, m\left|-\frac{J}{\Delta_{2}}\right| 2, m\right\rangle\left\langle 3, m\left|-\frac{J}{\Delta_{3}}\right| 3, m\right\rangle\left\langle 4, m\left|+\frac{J}{\Delta_{4}}\right| 4, m\right\rangle\langle 5, m|\right),
\end{aligned}
$$

where $\phi_{m, j}=\pi m / 2+\hat{k}_{j} \cdot \hat{r}-\delta_{R, j} t . \delta_{R, j}$ is chosen to be close to $\Delta_{j}$, but off-resonate to other $\Delta_{i}, i \neq j$ so that the Raman potential $V_{\text {Raman,j }}$ only couples $|j, m\rangle$ to its neighbor state $|j+1, m\rangle$.

Without Raman coupling, atoms in well $j$ are not coupled with other wells, yielding a state at $q=(j-1) 2 \pi /(5 d)$ of the lowest band of the superlattice

$$
\left|\psi_{q=(j-1) 2 \pi /(5 d)}^{(j)}\right\rangle=\sum_{m=1}^{N} \frac{1}{\sqrt{N}} e^{i \frac{2 \pi(j-1)}{5 d}\left[z_{m}+(j-1) \frac{d}{5}\right]}|j, m\rangle .
$$

Here $N$ is the number of unit cells in the superlattice.

The intra-band couplings

$$
\begin{aligned}
\left\langle\psi_{q=j 2 \pi /(5 d)}^{(j)}\left|V_{\text {Raman }, j}\right| \psi_{q=(j-1) 2 \pi /(5 d)}^{(j)}\right\rangle & =\sum_{m, m^{\prime}} \frac{1}{N} e^{i \frac{2 \pi}{5}\left[\left(m^{\prime}-m-\frac{1}{5}\right) j-m^{\prime}+\frac{1}{5}\right]}\left\langle j, m\left|V_{\text {Raman }, j}\right| j, m^{\prime}\right\rangle \\
& =\sum_{m} \frac{1}{N} e^{-i \frac{2 \pi}{5}\left[m+(j-1) \frac{1}{5}\right]}\left\langle j, m\left|V_{\text {Raman }, j}\right| j, m\right\rangle \\
\left\langle\psi_{q=(j-1) 2 \pi /(5 d)}^{(j+1)}\left|V_{\text {Raman }, j}\right| \psi_{q=j 2 \pi /(5 d)}^{(j+1)}\right\rangle & =\sum_{m, m^{\prime}} \frac{1}{N} e^{i \frac{2 \pi}{5}\left[\left(m^{\prime}-m-\frac{1}{5}\right) j-m\right]}\left\langle j+1, m\left|V_{\text {Raman }, j}\right| j+1, m^{\prime}\right\rangle \\
& =\sum_{m} \frac{1}{N} e^{-i \frac{2 \pi}{5}\left[m-\frac{j}{5}\right]}\left\langle j+1, m\left|V_{\text {Raman }, j}\right| j+1, m\right\rangle,
\end{aligned}
$$

and the SOC

$$
\begin{aligned}
\left\langle\psi_{q=j 2 \pi /(5 d)}^{(j+1)}\left|V_{\text {Raman }, j}\right| \psi_{q=(j-1) 2 \pi /(5 d)}^{(j)}\right\rangle & =\sum_{m, m^{\prime}} \frac{1}{N} e^{i \frac{2 \pi}{5}\left[\left(m^{\prime}-m-\frac{2}{5}\right) j-m^{\prime}+\frac{1}{5}\right]}\left\langle j+1, m\left|V_{\text {Raman }, j}\right| j, m^{\prime}\right\rangle \\
& =\sum_{m} \frac{1}{N} e^{-i \frac{2 \pi}{5}\left[m+(2 j-1) \frac{1}{5}\right]}\left\langle j+1, m\left|V_{\text {Raman }, j}\right| j, m\right\rangle .
\end{aligned}
$$

The intra-band terms cause a density modulation. If only near-resonant terms are kept, the single particle Hamiltonian can be written as

$$
\left(\begin{array}{ccccc}
\frac{\hat{p}^{2}}{2}+\Delta_{1}+\Delta_{2} & c_{p, 1} \frac{J}{\Delta_{1}} \Omega_{1} e^{-i\left(\hat{k}_{1} \cdot \hat{r}-\delta_{R, 1} t\right)} & 0 & 0 & 0 \\
c_{p, 1}^{*} \frac{J}{\Delta_{1}} \Omega_{1} e^{i\left(\hat{k}_{1} \cdot \hat{r}-\delta_{R, 1} t\right)} & \frac{\hat{p}^{2}}{2}+\Delta_{2} & c_{p, 2} \frac{J}{\Delta_{2}} \Omega_{2} e^{-i\left(\hat{k}_{2} \cdot \hat{r}-\delta_{R, 2} t\right)} & 0 & 0 \\
0 & c_{p, 2}^{*} \frac{J}{\Delta_{2}} \Omega_{2} e^{i\left(\hat{k}_{2} \cdot \hat{r}-\delta_{R, 2} t\right)} & \frac{\hat{p}^{2}}{2} & c_{p, 3} \frac{J}{\Delta_{3}} \Omega_{3} e^{-i\left(\hat{k}_{3} \cdot \hat{r}-\delta_{R, 3} t\right)} & 0 \\
0 & 0 & c_{p, 3}^{*} \frac{J}{\Delta_{3}} \Omega_{3} e^{i\left(\hat{k}_{3} \cdot \hat{r}-\delta_{R, 3} t\right)} & \frac{\hat{p}^{2}}{2}-\Delta_{3} & c_{p, 4} \frac{J}{\Delta_{4}} \Omega_{4} e^{-i\left(\hat{k}_{4} \cdot \hat{r}-\delta_{R, 4} t\right)} \\
0 & 0 & 0 & c_{p, 4}^{*} \frac{J}{\Delta_{4}} \Omega_{4} e^{i\left(\hat{k}_{4} \cdot \hat{r}-\delta_{R, 4} t\right)} & \frac{\hat{p}^{2}}{2}-\Delta_{3}-\Delta_{4}
\end{array}\right),
$$

where complex constants $c_{p, j}$ for each SOC terms are determined by Eqs. (10) and (14). A unitary transformation $U_{t}$ defined as $|1\rangle_{t} \rightarrow|1\rangle_{t} e^{-i\left(\delta_{R, 1}+\delta_{R, 2}\right) t},|2\rangle_{t} \rightarrow|2\rangle_{t} e^{-i \delta_{R, 2} t},|3\rangle_{t} \rightarrow|3\rangle_{t},|4\rangle_{t} \rightarrow|4\rangle_{t} e^{-i \delta_{R, 3} t},|5\rangle_{t} \rightarrow|5\rangle_{t} e^{-i\left(\delta_{R, 3}+\delta_{R, 4}\right) t}$ for each basis $|j\rangle_{t}$ can be applied to eliminate the time-dependency of SOC, yielding the effective Hamiltonian

$$
H_{5,0}=\sum_{j=1}^{5} \frac{\hat{p}^{2}}{2}|j\rangle\langle j|+\sum_{j=1}^{4}\left(\frac{\Omega}{2} e^{-2 i \hat{k}_{j} \cdot \hat{r}}|j\rangle\langle j+1|+\text { h.c. }\right) .
$$




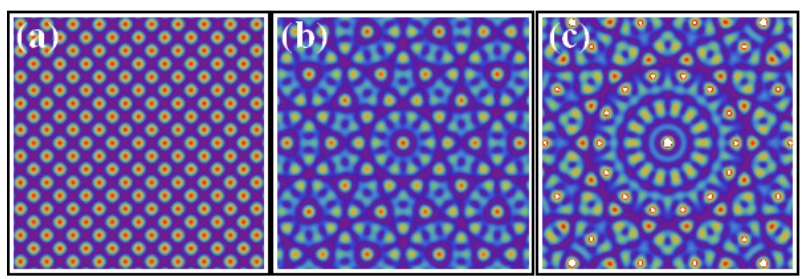

FIG. 6: (Color online) Crystalline and quasicrystal patterns in real-space when the minima in $k$-space form a regular polygon and are evenly populated. (a) $n=4$, namely, a square in momentum space. Discrete translational symmetries in both $x$ and $y$ directions are preserved. (b) and (c) correspond to $n=5$ (regular pentagon) and $n=7$ (heptagon) respectively. Discrete translational symmetries are broken and corresponding rotational symmetries are exhibited.

where the laser strengths are chosen such that each SOC term has the same effective Raman coupling strength $\Omega=J\left|c_{p, j}\right| \Omega_{j} / \Delta_{j}$. The detunings are canceled out for exactly resonant case.

\section{$k$-space configuration}

The spatial dependence of the Raman coupling can be removed by a unitary transformation

$$
|j\rangle \rightarrow e^{i \hat{i}_{j} \cdot \hat{r}}|j\rangle, \text { for } j=1 \ldots 5,
$$

for each state, where $\hat{l}_{j}$ is a constant vector. In this new pseudo-momentum basis, $\hat{l}_{j}$ must satisfy the following group of equations

$$
-\hat{l}_{j}-2 \hat{k}_{j}+\hat{l}_{j+1}=0, \text { for } j=1 \ldots 4 \text {. }
$$

to eliminate the spatial dependence of Raman couplings. However, these four equations are not sufficient to determine all variables. The fifth equation can be obtained from minimizing the single particle energy functional. Considering the simplest case $\Omega=0$ with the kinetic energy given by

$$
E_{k, 0}=\frac{1}{2} \sum_{j=1}^{5} \hat{l}_{j}^{2}=\frac{1}{2} \hat{l}_{1}^{2}+\sum_{j=2}^{5}\left(\hat{l}_{1}+2 \sum_{j-1}^{4} \hat{k}_{j}\right)^{2} .
$$

Minimizing this functional yields $\hat{l}_{1}=-\frac{2}{5} \sum_{j=1}^{4}(5-j) \hat{k}_{j} . \hat{k}_{j}$ is exactly the same as that in the main text for a pentagon in momentum space with one point fixed at $(0,1)$. Inserting $\hat{k}_{j}$ back, one finds $\hat{l}_{1}=(0,1)$, which is consistent with our configuration. Therefore $\hat{l}_{j}$ are nothing but the minima in $k$-space, that is, $\hat{l}_{j}=\hat{p}_{j}$. This is not true for arbitrary configuration but holds for any regular polygon. If all minima are occupied equally, we may have crystal or quasicrystal orders. In Fig. 6, we illustrate the spatial distribution patterns for typical crystal and quasicrystal orders. Panel (a) is a simple crystal with four band minima and discrete lattice translational symmetry. (b) [(c)] is a quasicrystal with 5 -fold (7-fold) rotational symmetry if the phase distribution is taken into account. Discrete lattice translational symmetry is obviously absent. For any $n \geq 7$, we may expect a quasicrystal structure in its spatial pattern.

\section{Non-zero-momentum plane-wave phase}

With interactions, the plane-wave phase would occur at a large Raman coupling. The Hamiltonian can be rewritten as

$$
H_{5,0}^{\prime}=\Omega\left(\frac{1}{\Omega} H_{p}+H_{s o c}\right),
$$

where $H_{p}=\sum_{j=1}^{n} \frac{1}{2}\left(p-p_{j}\right)^{2}|j\rangle\langle j|$ is the kinetic energy and $H_{s o c}=\sum_{j=1}^{n-1}(|j\rangle\langle j+1|+H . c$.$) . In the large \Omega$ region, the kinetic energy term can be treated as a perturbation. The first-order energy correction is

$$
E_{0}^{(1)}=\left\langle\Phi_{0}\left|\frac{1}{\Omega} H_{p}\right| \Phi_{0}\right\rangle=\frac{1}{2 \Omega} \sum_{j}\left|a_{j}\right|^{2}\left(p-p_{j}\right)^{2},
$$


where $\Phi_{0}=\sum_{j} a_{j}|j\rangle$ is the ground state of $H_{s o c}$ with the normalization $\sum_{j}\left|a_{j}\right|^{2}=1$. A simple variational method shows that the minimum locates at $\sum_{j}\left|a_{j}\right|^{2} p_{j}$, which is generally non-zero, yielding a non-zero-momentum plane-wave phase. 